\title{
Eb Meter Monitor And Control Using Internet of Things
}

\author{
*Rajarajeshwari.K.C ${ }^{1}$, Angel Antonette Keziah.J ${ }^{2}$ \\ ${ }^{1}$ Assistant Professor, Department of Electronics and communicaton Engineering ,Sri Ramakrishna Institute of \\ Technology, Coimbatore, India $^{I}$ \\ ${ }^{2}$ Assistant Professor ,Department of Electronics and communicaton Engineering ,Sri Ramakrishna Institute of \\ Technology, Coimbatore , India ${ }^{2}$
}

\begin{abstract}
The design and development of a smart monitoring and controlling system for household electrical appliances in real time is implemented. The system principally monitors electrical parameters of household appliances such as voltage and current and subsequently calculates the power consumed. The novelty of this system is the implementation of the controlling mechanism of appliances in different ways. The developed system is a low-cost and flexible in operation and thus can save electricity expense of the consumers. The prototype has been extensively tested in real-life situations and experimental results are very encouraging
\end{abstract}

Keywords: Energy efficient algorithm, Manet, total transmission energy, maximum number of hops, network lifetime

\section{Introduction}

The last meter smart grid is the portion of the smart grid closer to the home, and the one with which customers ,interact. It allows a two-way data flow between customers and electric utilities, transforming the "traditionally passive end-users into active players" in the energy market. Considering the seven domains of the conceptual model of smart grids proposed by the National Institute of Standards and Technology, the last-meter smart grid corresponds to the "customer domain." It enables residential, commercial, and industrial customersbased on their different energy needs - to optimize energy consumption and local generation, and to actively participate to demand-response policies, one of the most disrupting aspects of smart grids. Nontechnical customers need a simple way to control energy consumption and production, and to exchange power usage data at the proper level of granularity with energy providers or distributors. And the recent trend is when a user gets EB bill consecutively for two months and if the second month's bill is comparatively higher than that of the first, the EB department compares the two bill's and verify. When the EB department calculates the period in which the current has been used more than in the same period of the previous month, the user will receive the intimation such that they can reduce the usage accordingly.

\section{Literature Survey}

The necessity to promote smart grid (SG) has been recognized with a strong consensus. The SG integrates electrical grids and communication infrastructures and forms an intelligent electricity network working with all connected components to deliver sustainable electricity supplies. Many advanced communication technologies have been identified for SG applications with a potential to significantly enhance the overall efficiency of power grids. In this paper, the challenges and applications of communication technologies in SG are discussed. In particular, we identify three major challenges to implement SG communication systems, including standards interoperability, cognitive access to unlicensed radio spectra, and cyber security. The issues to implement SG communications on an evolutional path and its future trends are also addressed. The aim of this paper is to offer a comprehensive review of state-of-the-art researches on SG communications[1].

Smart grid aims to empower the current power grid with the capability of supporting two-way energy and information flow; and facilitating the integration of advanced computer technology and renewable energy sources into the grid. We assume a smart home with a wireless sensor network based on Zigbee . The smart home contains elements like light or temperature sensors at every room, HVAC (heating, ventilation, and air conditioning), smart appliances, thermostat and smart meter. Furthermore, there is a central computer that can communicate with all these elements. Web service is implemented on central computer and it can be accessed over the internet. The paper proposes an approach that makes use of the web services technologies to remotely interact with smart home elements in a smart grid environment. These interactions include adjusting the temperature according to personal preference or reading energy consumption. Furthermore, utility provider can interact with the smart home via web services and can facilitate demand response or selling energy back to the grid. Some scenarios are shown to describe the interactions in more detail[2]. 
Energy management means to optimize one of the most complex and important technical creations that we know: the energy system. While there is plenty of experience in optimizing energy generation and distribution, it is the demand side that receives increasing attention by research and industry. Demand Side Management (DSM) is a portfolio of measures to improve the energy system at the side of consumption. It ranges from improving energy efficiency by using better materials, over smart energy tariffs with incentives for certain consumption patterns, up to sophisticated real-time control of distributed energy resources. This paper gives an overview and a taxonomy for DSM, analyzes the various types of DSM, and gives an outlook on the latest demonstration projects in this domain[3].

The recent worldwide measures for energy savings call for a larger awareness of the household energy consumption, given the relevant contribution of domestic load to the national energy balance. On the other hand, electricity smart meters together with gas, heat, and water meters can be interconnected in a large network offering a potential value to implement energy savings and other energy-related services, as long as an efficient interface with the final user is implemented. Unfortunately, so far, the interface of such devices is mostly designed and addressed at the utilities supervising the system, giving them relevant advantages, while the communication with the household is often underestimated. This paper addresses this topic by proposing the definition of a local interface for smart meters, by looking at the actual European Union and international regulations, at the technological solutions available on the market, and at those implemented in different countries, and, finally, by proposing specific architectures for a proper consumer-oriented implementation of a smart meter network[4].

\subsection{Design Considerations}

\section{Proposed Work}

- High Precision

- Long Life

- Low Power Consumption

- Running Stably

- Anti-tamper

- Automatic Meter-reading

- Running Stably

\subsection{Description of the Proposed Method}

3.2.1Energy meter

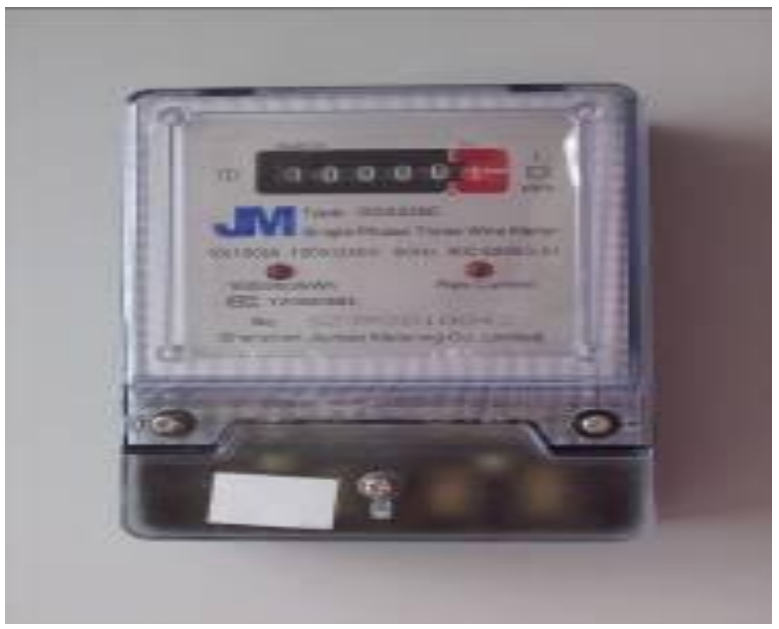

Fig.1 Energy meter

High Precision: The watt-hour meter is fully electronic type design, with core of imported dedicated energy measuring chip, assuring the accurate measurement, and the precision is impregnable against the frequency, voltage, ultra harmonics, and temperature.

Long Life: Adopt the SMT manufacture technique, applying the international well-known brand, long life components; assure the usage life more than 10 years.

Low Power Consumption: Adopt the optimum design, its own power consumption is less than or equal to $0.8 \mathrm{~W}$. When used in a wide range, it's available to reduce the consumption of power grid, increase the efficiency of electricity supply. 
Running Stably: Possesses good anti-interference performance, strong overload capacity, wide working range; make the watt-hour meter run stably.

Anti-tamper: Be able to prevent such electric larceny ways as reverse wire connection, plus magnet, sloped installation, etc.

Power Cut Control: Built-in magnet latching relay, it accomplishes meter code setting, meter-reading and switch on/off controlling of relay through RS485. (Optional)

Automatic Meter-reading: Possesses RS485, infrared and impulse output port, available for remote meter reading, while the watt-hour meter receive the collector's meter reading instruction, return to collector the current accumulated electric use quantity and meter-reading electric use quantity from last time to current.

\subsubsection{Internet Of Things}

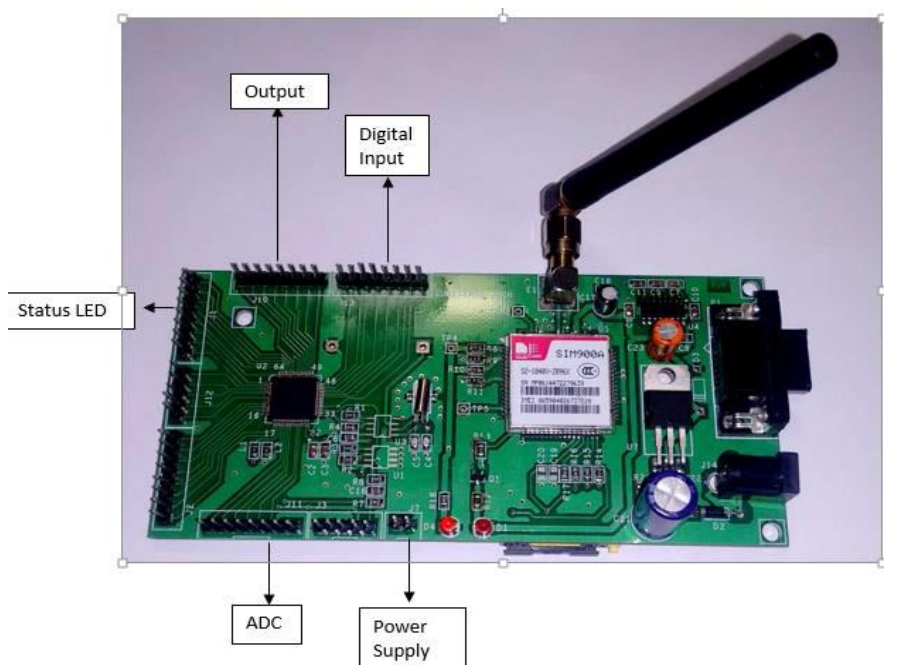

Fig.2 IOT Kit

The Internet of Things (IOT) is an environment in which objects, animals or people are provided with unique identifiers and the ability to transfer data over a network without requiring human-to-human or humanto-computer interaction. IoT has evolved from the convergence of wireless technologies, microelectromechanical systems (MEMS) and the Internet. The concept may also be referred to as the Internet of Everything. A thing in the Internet of Things, can be a person with a heart monitor implant, a farm animal with a biochip transponder, an automobile that has built-in sensors to alert the driver when tire pressure is low or any other natural or man-made object that can be assigned an IP address and provided with the ability to transfer data over a network.

\subsubsection{Peripheral Interface Controller $16 f 877$}

The microcontroller that has been used for this project is from PIC series. PIC microcontroller is the first RISC based microcontroller fabricated in CMOS (complimentary metal oxide semiconductor) that uses separate bus for instruction and data allowing simultaneous access of program and data memory. The main advantage of CMOS and RISC combination is low power consumption resulting in a very small chip size with a small pin count. The main advantage of CMOS is that it has immunity to noise than other fabrication techniques. Various microcontrollers offer different kinds of memories. EEPROM, EPROM, FLASH etc. are some of the memories of which FLASH is the most recently developed. Technology that is used in pic16F877 is flash technology, so that data is retained even when the power is switched

\subsubsection{Liquid Crystal Display}

LCD (Liquid Crystal Display) screen is an electronic display module and find a wide range of applications. A $16 \times 2$ LCD display is very basic module and is very commonly used in various devices and circuits. These modules are preferred over and other multi segment $\mathrm{s}$. The reasons being: LCDs are economical; easily programmable; have no limitation of displaying special \& even (unlike in seven segments), and so on. $16 \times 2$ LCD means it can display 16 characters per line and there are 2 such lines. In this LCD each character is displayed in $5 \times 7$ pixel matrix. This LCD has two registers, namely, Command and Data. 


\subsection{4buzzer}

A buzzer or beeper is a signalling device, usually electronic, typically used in automobiles, household appliances such as a microwave oven, or game shows. It most commonly consists of a number of switches or sensors connected to a control unit that determines if and which button was pushed or a preset time has lapsed, and usually illuminates a light on theappropriate button or control panel, and sounds a warning in the form of a continuous or intermittent buzzing or beeping sound. Initially this device was based on an electromechanical system which was identical to an electric bell without the metal gong (which makes the ringing noise). Often these units were anchored to a wall or ceiling and used the ceiling or wall as a sounding board. Nowadays, it is more popular to use a ceramic-based piezoelectric sounder like a Son alert which makes a high-pitched tone.

\subsubsection{Relay}

A relay is an electrical switch that opens and closes under the control of another electrical circuit. In the original form, the switch is operated by an electromagnet to open or close one or many sets of contacts. It was invented by Joseph Henry in 1835. Because a relay is able to control an output circuit of higher power than the input circuit, it can be considered to be, in a broad sense, a form of an electrical amplifier.

\subsubsection{Embedded C}

Embedded $\mathrm{c}$ is a set of language extensions for the address commonality issues that exist between $\mathrm{C}$ extensions for different. Historically, embedded $\mathrm{C}$ programming requires nonstandard extensions to the $\mathrm{C}$ language in order to support exotic features such as operations. In 2008, the C Standards Committee extended the $\mathrm{C}$ language to address these issues by providing a common standard for all implementations to adhere to. It includes a number of features not available in normal $\mathrm{C}$, such as, fixed-point arithmetic, named address spaces, and basic I/O hardware addressing.Embedded $\mathrm{C}$ use most of the syntax and semantics of standards $\mathrm{C}$, e.g., main() function, variable definition, data type declaration, conditional statements (if, switch. case), loops (while, for), functions, arrays and strings, structures and union, bit operations, macros, unions, etc. The C programming language is perhaps the most popular programming language for programming embeddedsystems.

\subsection{Software Output}

\section{Simulation Results}

To ensure a high-quality product, diagrams and lettering MUST be either computer-drafted or drawn using The simulation studies involve the deterministic small network topology with 5 nodes as shown in Fig.3.

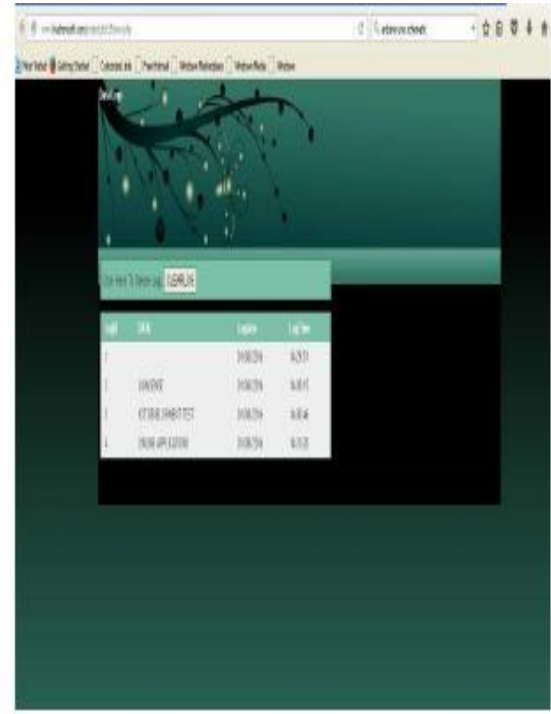

Fig.3 Simulation Output

In this there are two webpages one webpage is to check the electrical units consumed for a particular time delay. Second webpage is to disconnect the load if the electrical bill is not paid by the customers. The webpage is operated using the IOT. These webpages are kept confidentially, it has a clear log option to clear the previous data. 


\subsection{Hardware Output}

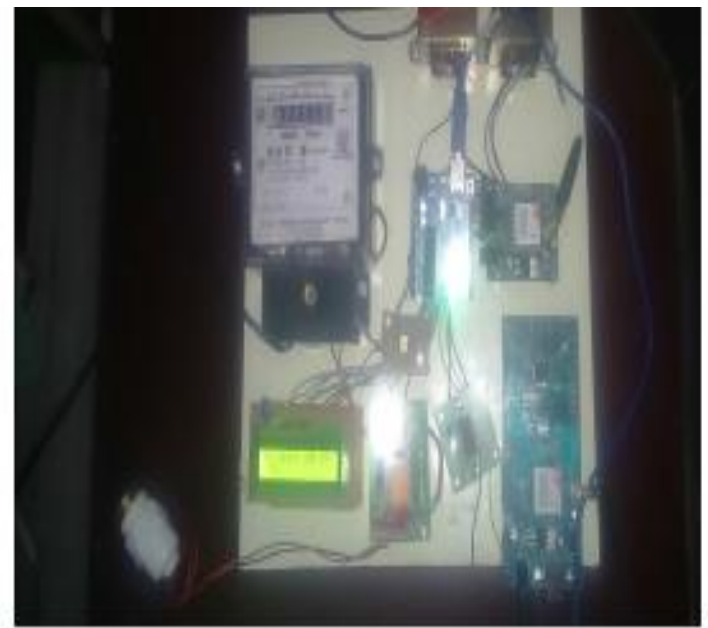

Fig.4 Hardware output

In the hardware output the unit consumed is displayed in the LCD display. It has IOT by which the notification regarding the electricity consumption is send to the webpage of the customer. The load is disconnected using the relay by the webpage.

\section{Conclusion}

The design and development of a smart monitoring and controlling system for household electrical appliances in real time is implemented. The system principally monitors electrical parameters of household appliances such as voltage and current and subsequently calculates the power consumed. The developed system is a low-cost and flexible in operation and thus can save electricity expense of the consumers. The recent trend is when a user gets EB bill consecutively for two months and if the second month's bill is comparatively higher than that of the first, the EB department compares the two bill's and verify. when the EB department calculates the period in which the current has been used more than in the same period of the previous month, the user will receive the intimation such that theycan reduce the usage accordingly.

\section{References}

[1] R. Ma, H. H. Chen, Y. Huang, and W. Meng "Smart grid communication: Its challenges and opportunities," IEEE Trans. Smart Grid, vol. 4, no. 1, pp. 36-46, Mar. 2013

[2] A. Khan and H. T. Mouftah, "Web services for indoor energy management in a smart grid environment," in Proc. 2011 IEEE 22 nd Int. Symp . Pers. Indoor Mobile Radio Communication. (PIMRC), pp. 1036-1040.

[3] P. Palensky and D. Dietrich, "Demand side management : Demand response, intelligent energy systems, and smart loads," IEEE Trans. Ind. Informat., vol. 7, no. 3, pp. 381- 388, Aug. 2011

[4] F. Benzi, N. Anglani, E. Bassi, and L. Frosini, "Electricity smart meters interfacing the households," IEEE Trans. Ind. Electron., 2011

\section{Biography}

[1]. RajaRajeshwari K.C is an Assistant Professor in the Electronics and communication engineering Department, Sri Ramakrishna Institute of Technology, Coimbatore. She received Master of Engineering (ME) degree in 2016 from Sri Ramakrishna Institute of Technology, Coimbatore, India. Her research interests are Antennas, IoT.

[2]. Angel Antonette Keziah.J is an Assistant Professor in the Electronics and communication engineering Department, Sri Ramakrishna Institute of Technology, Coimbatore. She received Master of Engineering (ME) degree in 2017 from PSG College of Technology, Coimbatore, India. Her research interests are Communication, networking, IoT.

IOSR Journal of Electronics and Communication Engineering (IOSR-JECE) is UGC approved Journal with Sl. No. 5016, Journal no. 49082.

Rajarajeshwari. K.C. "Eb Meter Monitor And Control Using Internet of Things." IOSR Journal of Electronics and Communication Engineering (IOSR-JECE) 12.4 (2017): 9-13. 